\title{
Multi-TOA Based Position Estimation for IR-UWB
}

\author{
Genís Floriach*, Montse Nájar* and Monica Navarro $^{\dagger}$ \\ ${ }^{*}$ Department of Signal Theory and Communications \\ Universitat Politècnica de Catalunya (UPC), Barcelona, Spain \\ ${ }^{\dagger}$ Centre Tecnològic de Telecomunicacions de Catalunya (CTTC) \\ Castelldefels, Barcelona, Spain \\ Emails: genis.floriach@alu-etsetb.upc.edu, montse.najar@upc.edu, monica.navarro@cttc.es
}

\begin{abstract}
The problem of localizing an IR-UWB transmitter from the signals received at several anchors is considered. The positioning problem is typically solved in a two-step approach where in the first step the Time of Arrival (TOA) is estimated independently at each anchor, and the position estimate is found in a second step. However, this approach can be improved, especially in challenging scenarios, if the positioning problem is treat as a whole, that is, the target position is estimated directly from the signals received on each anchor (Direct Position estimation DPE). In this paper, we present a different approach that sits halfway between these two approaches. The algorithm is based on a soft two-steps approach, where several possible TOA estimators are selected in the first step, and then the best estimators are used to find the position. The performance of the method is assessed under the framework of the IEEE 802.15.4a channel models.
\end{abstract}

\section{INTRODUCTION}

Recently, UWB (Ultra Wide Band) technology [1], [2] has drawn attention due to its precise localization capabilities. Specially in indoor wireless environment, where the dense multipath makes position estimation a very challenging task. The use of extremely short time domain pulses with several $\mathrm{GHz}$ of bandwidth offers a high time resolution which allows resolving the multipath components as well as penetrating obstacles.

To provide accurate position information of nodes, signals are exchanged between the node and a number of reference nodes ("Anchor" nodes) whose positions are known. The position of a target node can be estimated by the target node itself (self-positioning), or it can be estimated by a central unit that gathers position information from the reference nodes (remote-positioning).

There are several algorithmic approaches to wireless location with various degrees of precision and accuracy. In general, triangulation algorithms that combine the distances estimated between different reference nodes and the target to be located are applied. This procedure is the so called two-steps approach. Depending on the algorithm, distance measurements are based on different signal parameters being the delay or Time of Arrival (TOA) the most suitable for UWB systems as it takes advantage of the high time resolution of UWB signals [1],

This work has been supported by the Spanish Ministry of Economy and Competitiveness (Ministerio de Economía y Competitividad) through project TEC2016-77148-C2-1-R (RUNNER), and from the Catalan Government (AGAUR) through grant 2014 SGR 60.
[3], [4]. However, as proved in [5], the two-step procedures are suboptimal because in the first stage, the measurements at distinct anchors are independent and ignore the constraint that all measurements must be consistent with a single emitter. Therefore, we can achieve better performance, especially in challenging scenarios, if we merge the two positioning steps into a single stage and then estimate the target position directly from the signals received on each anchor node (Direct position estimation DPE). Nonetheless, the improved accuracy of DPE comes at cost of higher complexity and computational load as well as the requirement of transmitting the whole signal to a central processing node.

The Cramer-Rao lower bounds of DPE and two-step for the single-path model have been developed in [5]. The analytical lower bounds prove that DPE attains lower variance than twostep. However, the single-path model is not appropriate for UWB channels as it presents many multipath components. The Maximum-likelihood (ML) for dense multipath channels is known [4], however, it is too complex for practical implementation. That is why many proposals aim for a simplified multipath model. The authors in [6] propose the use of signal classification (MUSIC) method and focusing matrices. However, it assumes that the number of multipath reflectors is known and that they are smaller that the number of receivers. In [7] the authors developed and approximation of the ML for dense multipath channels by treating the received signal as Gaussian. Recent works [8] suggest the use of the ExpectationMaximization algorithm to find the Maximum Likelihood, however, it has not been tested in dense multipath scenarios like the ones we find in UWB.

In this paper, we revisit the DPE approach presented by the authors in [9] and propose a novel technique to overcome the drawbacks present in this approach. The proposed algorithm, unlike the DPE approach, does not work with the complete received signal but instead it works with a set of measurements or possible TOA estimators, all these measurements are later used to determine the node position. Consequently, it reduces the ambiguity in the selection of the proper peak present in all Threshold-based TOA estimators. Moreover, the amount of data required to be transmitted to the central node is much lower and the computational complexity is reduced as well.

The rest of the paper is organized as follows: Section II introduces the IR-UWB signal model, Section III and 
IV reviews the two-step positioning scheme based on TOA estimates and DPE scheme proposed by the authors in [3] and [9] respectively. The proposed Multi-TOA approach is introduced in Section V. Performance evaluation is given in Section VI and conclusions are drawn in VII.

\section{IR-UWB SYSTEM MODEL}

The IR-UWB signal model is considered to be a train of unit energy gaussian pulses $p(t)$ of very short duration that propagates through an $M$-path tap delay channel. The channel impulse response is $\sum_{m=0}^{M-1} h_{m} \delta\left(t-\tau_{m}\right)$, with $\tau_{0}<\tau_{1}<$ $\ldots<\tau_{M-1}$, being $\tau_{0}$ the TOA that has to be estimated. The received signal is then expressed as:

$$
y(t)=\sum_{m=0}^{M-1} \sum_{k=-\infty}^{\infty} h_{m} p\left(t-k T_{f}-\tau_{m}\right)+w(t)
$$

where $T_{f}$ is the pulse repetition period also referred to as frame period and $w(t)$ is zero-mean white noise with variance $\sigma^{2}$. The signal associated to the $k$-th transmitted pulse, in the frequency domain is given by:

$$
Y_{k}(\omega)=\sum_{m=0}^{M-1} h_{m} S_{k}(\omega) e^{-j w \tau_{m}}+V_{k}(\omega)
$$

The frequency component associated to the shifted pulse is given by $S_{k}(\omega)=P(\omega) e^{-j \omega k T_{f}}$, with $P(\omega)$ being the Fourier Transform of the pulse $p(t)$ and $V_{k}(\omega)$ is the noise associated to the $k$-th symbol. Sampling (2) at $\omega_{n}=\omega_{0} n$ for $n=0,1, \ldots, N-1$ where $\omega_{0}=\frac{2 \pi}{N}$, rearranging the frequency domain samples $Y_{k}[n]$ into the vector $\mathbf{Y}_{k} \in \mathbb{C}^{N \times 1}$ and explicitly separating the LOS term yields

$$
\mathbf{Y}_{k}=h_{0} \mathbf{S}_{k} \mathbf{e}_{\tau_{0}}+\tilde{\mathbf{V}}_{k}
$$

where $\tilde{\mathbf{V}}_{k}=\mathbf{S}_{k} \mathbf{E}_{\tau} \mathbf{h}+\mathbf{V}_{k}$ and $\mathbf{S}_{k} \in \mathbb{C}^{N \times N}$ is a diagonal matrix whose components are the frequency samples of $S_{k}(\omega)$. The matrix $\mathbf{E}_{\tau} \in \mathbb{C}^{N \times M-1}$ contains the delay signature vectors associated to each arriving delayed signal of the multipath,

$$
\mathbf{E}_{\tau}=\left[\begin{array}{lllll}
\mathbf{e}_{\tau_{1}} & \ldots & \mathbf{e}_{\tau_{m}} & \ldots & \mathbf{e}_{\tau_{M-1}}
\end{array}\right]
$$

with $\mathbf{e}_{\tau_{m}}=\left[\begin{array}{llll}1 & e^{-j \omega_{0} \tau_{m}} & \ldots & e^{-j \omega_{0}(N-1) \tau_{m}}\end{array}\right]^{T}$. The channel fading coefficients, except for $h_{0}$ are arranged in the vector $\mathbf{h}=\left[\begin{array}{lll}h_{0} & \ldots & h_{M-1}\end{array}\right]^{T} \in \mathbb{R}^{M-1 \times 1}$, and the noise samples in vector $\mathbf{V}_{k} \in \mathbb{C}^{N \times 1}$.

\section{TOA-BASED POSITION ESTIMATION FOR IR-UWB}

Next, we review the TOA-based positioning scheme for IRUWB systems proposed by the authors in [3]. The estimator presented in this section is the basis for the more sophisticated estimators that will be presented in Section IV and V.

In this case, the position estimate is obtained by first measuring the distance to the anchor nodes at known locations and then performing a trilateration. To measure the distance, we consider the TOA estimation algorithm proposed by the authors in [3]. This estimator is based on calculating the pseudo-periodogram or power delay profile, defined as the signal energy distribution with respect to propagation delays. Namely,

$$
P_{\ell}(\tau)=\mathbf{e}_{\tau}^{H} \mathbf{R}_{\ell} \mathbf{e}_{\tau}
$$

where $\ell=1, \ldots, N_{A}$ denotes the anchor index, the superscript $(.)^{H}$ denotes the transpose complex conjugate, and $\mathbf{R}_{\ell}$ is the sample covariance matrix defined as,

$$
\mathbf{R}_{\ell}=\frac{1}{N_{f}} \sum_{k=1}^{N_{f}} \mathbf{Y}_{k}^{(\ell)}\left(\mathbf{Y}_{k}^{(\ell)}\right)^{H}
$$

where $N_{f}$ is the number of observed frames. The TOA estimate is the first peak in the periodogram $P_{\ell}$ that exceeds a certain threshold $\gamma$. The selection of the threshold is always a critical factor in all TOA estimation techniques based on threshold decisions. There are some proposals for the estimation of threshold values for specific channels [10], [11]. However, most of the proposals require some calibration and heuristic adjustment of some of the parameters that define the threshold level.

\section{Direct Position estimation FOR IR-UWB}

Here, we briefly review the DPE approach for IR-UWB [9] considered for baseline performance.

In this case, the positioning problem is solved on a single step performing a search directly over the spatial coordinates. Defining $\mathbf{Y}=\left(\mathbf{Y}^{(1)}, \ldots, \mathbf{Y}^{(\ell)}, \ldots, \mathbf{Y}^{\left(N_{A}\right)}\right)^{T}$, with $\mathbf{Y}^{(\ell)}=$ $h_{0, \ell} \mathbf{S e}_{\tau_{0, \ell}}+\tilde{\mathbf{V}}^{(\ell)}$ according to (3) (symbols index are dropped in order to ease notation), the system model can be written in terms of LOS contribution as,

$$
\mathbf{Y}=\overline{\mathbf{S}}_{0} \mathbf{e}_{\mathbf{p}}+\tilde{\mathbf{V}}
$$

where $\overline{\mathbf{S}}_{0}=\operatorname{diag}\left(h_{0,1} \mathbf{S}, \ldots, h_{0, N_{A}} \mathbf{S}\right)$ is a block diagonal matrix with pulse spectral components weighted by the LOS channel fading coefficients, $\mathbf{e}_{\mathbf{p}}=\left(\mathbf{e}_{f_{1}(\mathbf{p})}, \ldots, \mathbf{e}_{f_{N_{A}}(\mathbf{p})}\right)^{T}$ is the delay signature vector as a function of the target spatial coordinates $\mathbf{p}=[x, y]^{T}$, and $\tilde{\mathbf{V}}=\left(\tilde{\mathbf{V}}^{(1)}, \ldots, \tilde{\mathbf{V}}^{\left(N_{A}\right)}\right)^{T}$. The delay signature vectors $\mathbf{e}_{f_{\ell}(\mathbf{p})}$ are defined as in (4) but in this case the delay is related to the position vector $\mathbf{p}$ by the geometrical relation $f_{\ell}(\mathbf{p})=\left\|\mathbf{p}-\mathbf{p}_{\ell}\right\| / c$, with $\mathbf{p}_{\ell}$ being the two-dimensional coordinates of the $\ell$-th anchor. The DPE position estimate is the position vector that maximizes the following function,

$$
\hat{\mathbf{p}}=\underset{\mathbf{p}}{\operatorname{argmax}} \mathbf{e}_{\mathbf{p}}^{H} \mathbf{R e}_{\mathbf{p}}
$$

where $\mathbf{R}=\mathbf{Y} \mathbf{Y}^{H}$. Intuitively what this algorithm does is represent the periodograms in terms of the spatial coordinates and then add them up. We refer the reader to [9] for further information about this approach.

Although DPE techniques are expected to provide better results than the pure two-step approach, the proposed DPE in this section is still far from the optimum. The main sources of errors in the DPE come from the fact that UWB channels are not classical LOS scenarios where the LOS is present with stronger amplitude than other multipath components. In fact, 
there are channel realizations where delayed multipaths are considerably larger than the LOS.

As the periodograms estimated in each anchor are not functions to be maximized but instead we are interested in the first peak, it is not optimal to combine the signals adding them because then we lose the idea that first peaks are more important than strong peaks. To solve this problem we proposed a new approach based in Multi-TOA estimation.

\section{MUlti-TOA BASED POSITION ESTIMATION FOR IR-UWB}

Selection of the threshold is always a critical factor that may drop the performance drastically if it is not chosen properly. Then, the proposed solution is the following: instead of setting a threshold and keeping the first peak above it, all prominent peaks are selected and then all potential TOAs are used to find the position. All the potential TOAs are gathered in the central processing unit and the combination of TOAs that minimize the trilateration cost function are the ones selected. In other words, the position estimate will be the one that minimizes the following cost function:

$$
\hat{\mathbf{p}}=\underset{\mathbf{p}}{\operatorname{argmin}}\left[\min _{\mathbf{k}} \sum_{\ell=1}^{N_{A}}\left(\tau_{\ell}\left[k_{\ell}\right] c-\left\|\mathbf{p}-\mathbf{p}_{\ell}\right\|\right)^{2}\right]
$$

where $\mathbf{k}=\left[\begin{array}{lllll}k_{1} & \ldots & k_{\ell} & \ldots & k_{N_{A}}\end{array}\right] k_{\ell} \in\left\{1, \ldots, m_{\ell}\right\}, m_{\ell}$ is the number of peaks detected in the $\ell$-th anchor node and $\tau_{\ell}\left[k_{\ell}\right]$ is the potential $k_{\ell}$-th TOA detected in the $\ell$-th anchor node.

However, not all selected peaks are equally likely to be the true TOA. For example in the Fig. 1, the last peak to be the true TOA would mean that all the previous peaks were noise or pulse sidelobes of that peak and such thing is highly unlikely. Somehow, we must add information of the energy of the peak and the relative position of the peaks in the periodogram. Therefore, it is convenient to weight each peak by the probability of that peak being the true TOA. By weighting the peaks we improve the overall performance of the position estimation technique because the number of peaks gets reduced (last peaks will have probability 0 so we remove them) so it will be easier to minimize the cost function, and moreover we reduce the probability of choosing paths that do not represent the position of the target.

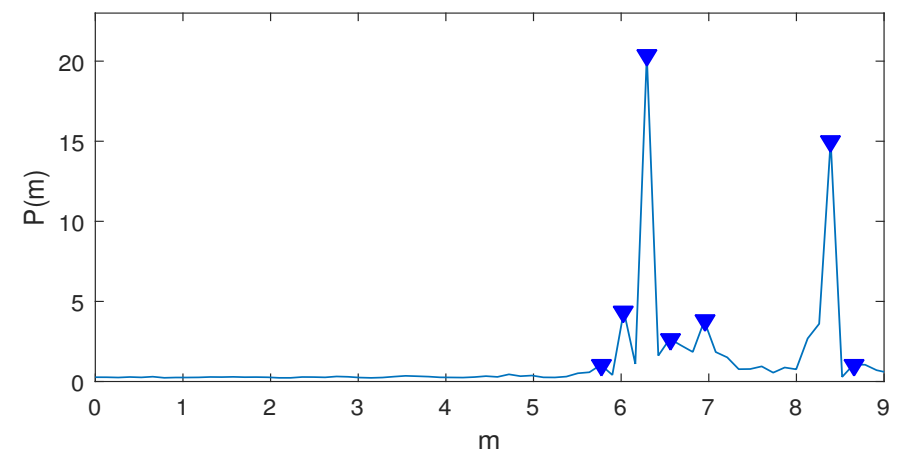

Fig. 1: Periodogram realization
However, as it happened with threshold selection (in the pure TOA periodogram approach), finding these probabilities is not an easy task. Then, the proposed weighting criterion is a heuristic approach based on the distribution of the energy of the TOA,

Weight $_{\text {peak }}=\prod_{k=0}^{i-1}\left(1-\right.$ Weight $\left._{\text {peak }}\right) \cdot P\left(E_{\text {TOA }}<E_{\text {peak }}\right)$

where $E_{T O A}$ is the random variable that represents the energy of the TOA (its distribution has been found empirically), Weight peak $_{0}=0$ and $E_{\text {peak }_{i}}$ is the Energy of a $i$ th detected peak. Finally, the position estimate is given by,

$$
\begin{array}{r}
\hat{\mathbf{p}}=\underset{\mathbf{p}}{\operatorname{argmin}}\left[\min _{\mathbf{k}} \sum_{\ell=1}^{N_{A}}\left(\tau_{\ell}\left[k_{\ell}\right] c-\left\|\mathbf{p}-\mathbf{p}_{\ell}\right\|\right)^{2}+\right. \\
\left.\lambda\left(-\sum_{\ell=1}^{N_{A}} W_{\text {eight }}\left[k_{\ell}\right]\right)\right]
\end{array}
$$

where $W e i g h t_{\ell}\left[k_{\ell}\right]$ is the weight of the $k_{\ell}$ peak of the $\ell$ th beacon and the parameter $\lambda$ is a trade-off between consistency and probability of the delays.

By using this approach, the problematic of strong multipath components present in the DPE is mitigated because now we take into account the fact that earlier peaks are more important than stronger peaks and the fact that peaks should be consistent. Moreover, it solves the ambiguity in the proper selection of the peak (present in the two steps approach) because several peaks are selected. In addition, whereas the DPE approach requires transmitting the whole signal to the central processing node, the Multi-TOA solution just requires transmitting the potential TOAs, which involves far less data.

\section{A. Implementation}

When it comes to solving the cost function in (11) we must deal with a function that depends on discrete variables (the variable $\mathbf{k}$ ). Addressing this discrete optimization problem by brute force, which is enumerating all the possible combinations, is not feasible as the number of combinations grows exponentially with the number of anchor nodes. Therefore, we propose the use of Branch and Bound [12] algorithm to try to reduce the search space.

Branch and Bound is a tree-search based algorithm where each node of the tree is a subproblem, then after solving a subproblem we can set a bound about how good the final solution (leaves of the tree) could potentially be. If that bound is better than an already found solution we have to expand this node and explore its children. Otherwise, we can prune that node, thus reducing the search space.

From this point onwards, we will refer to the anchor nodes as beacons and the nodes of a tree as nodes.

In our particular problem, the elements of the tree would be:

- Nodes: A particular trilateration using fewer beacons than the available. The depth of the tree is equal to the number of beacons 
- Lower Bound: The cost of that particular trilateration.

- Children: The children of a given parent node are the trilaterations using the same parameters of the parent node but adding the TOA information of the new beacon (the number of children is equal to the number of potential TOAs estimated by the new beacon).

- Leaves: A possible solution of the problem. There are as many leaves as number of combinations.

The way to explore the tree is a key factor to find the solution quickly. The proposed technique is the best-first search strategy which explores the tree by expanding the most promising node. It is important to note that although the worst case complexity of Branch and Bound is exponential, in practice, for our case of study it works well.

Algorithm 1 is a pseucode implementation of the proposed algorithm, where the Priority queue is a queue where each element has an associated priority (in our case the lower the bound the higher the priority), then an element with high priority is served before an element with low priority, and TreeNode is an structure that stores the beacons used, the potential TOA used for each beacon, the value of the bound and the estimated position if only these beacons were available.

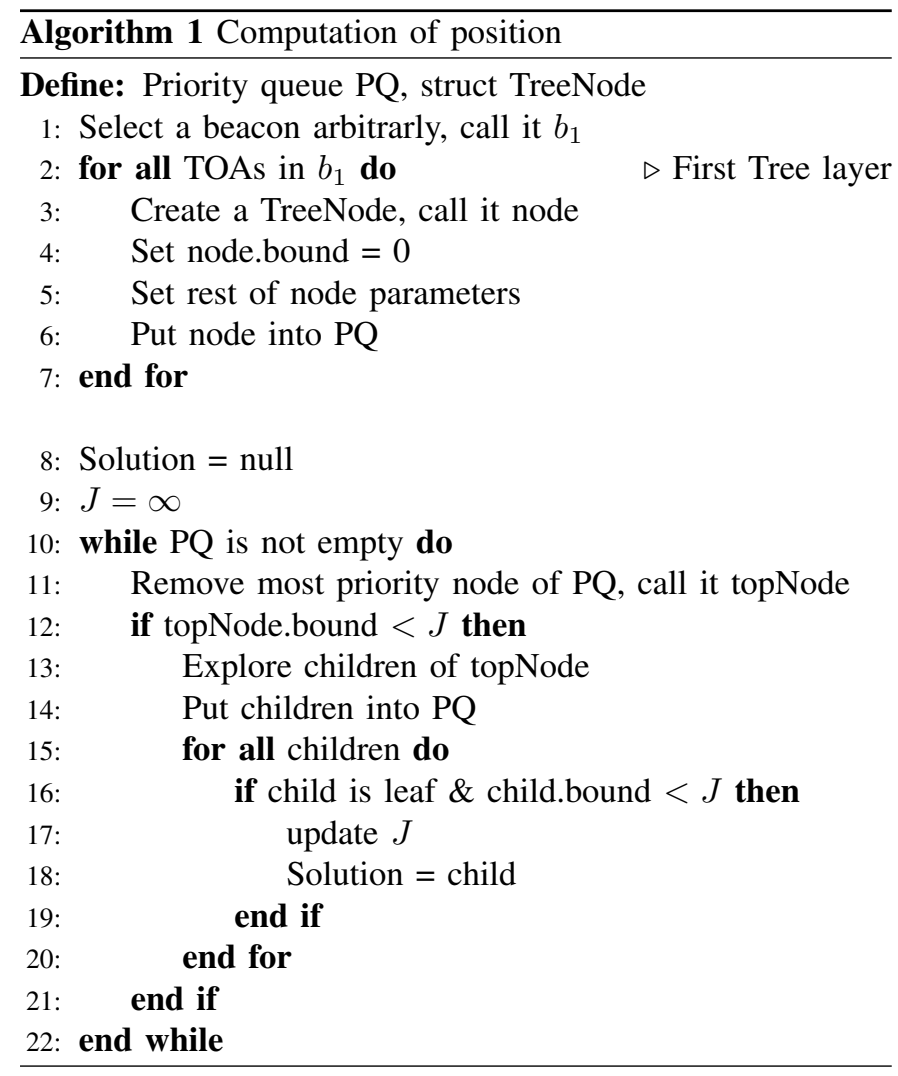

\section{NumericAl Results}

In this section, the performance of the proposed method is evaluated by means of numerical simulations with the IEEE 802.15.4a UWB channel models [13]. In particular, we focus on the CM3 office LOS and CM4 Office NLOS models which are indoor office environments characterized to exhibit dense multipath. The simulation setup is configured as follows: A single target is placed within a square room of $6 \times 6 \mathrm{~m}^{2}$ and is continuosly transmitting gaussian monocycle pulses of duration $T_{p}=1 \mathrm{~ns}$. The transmitted pulses are received by $N_{A}=4$ beacons placed at the corners of the room. The received signal is low pass filtered to avoid aliasing and sampled at $2 \mathrm{GHz}$. In all scenarios, the signal to noise ratio (SNR) at each beacon is set to $4 \mathrm{~dB}$. It is important to mention, however, that similar results were obtained for SNRs down to $-2 \mathrm{~dB}$.

Fig. 2a and Fig. 2b depicts the cost function (8) and (11) obtained for the DPE and Multi-TOA respectively in a particular realization for the CM3 Office LOS scenario. As mentioned before, in UWB channels the LOS can be attenuated with respect to multipath components. This fact can lead the DPE approach to estimate the position erroneously as we can see in Fig. 2a. However, the Multi-TOA approach does not suffer from such channel propagation feature, and manages to estimate the position properly.

For the evaluation of the position estimation accuracy, we considered 500 channel realizations for each scenario. The position error of DPE, Multi-TOA and TOA-based two-step approach is depicted in Fig. 3 in terms of the cumulative distribution function (CDF) for Office LOS (CM3) and Office NLOS (CM4) scenarios. The parameter $\lambda$ in (11) for the Multi-TOA approach is obtained empirically. In Fig. 3a there is also depicted the CDF for the DPE when using strictly LOS channels, that is, channels where the LOS is always the one with higher energy. In this case we can see that the performance is similar to the other approches. Such disparity between the two DPE curves shows that it is not robust to the strong attenuation of the LOS, this fact is emphasized in the Fig. $3 b$ where DPE performs extremely poorly. Fig.3a also shows the CDF for the two-steps when the threshold is deviated a $30 \%$ and a $40 \%$ from the optimum value. These curves show the sensibility of the two-step to the threshold value. From Fig. 3 it can be concluded that the proposed MultiTOA outperforms the DPE approach. Comparing Fig. 3a and Fig. $3 \mathrm{~b}$ it can also be concluded that the proposed algorithm are robust to the NLOS propagation impairments.

\section{CONCLUSIONS}

A novel position estimator for IR-UWB localization has been introduced and assessed under realistic channel models developed by the IEEE 802.15.4a standardization group. The proposed algorithm reduces the complexity of DPE approaches, since it does not require the sampled received signal but only the set of measurements (TOA estimates). Such complexity reduction does not incur in information loss for the positioning problem. Moreover, we show by means of numerical results that the proposed scheme performs better in terms of position estimate accuracy and is robust to NLOS propagation. Further work will focus on an analytical analysis of the proper weighting as well as an accurate analysis of the complexity of the proposed algorithm. 


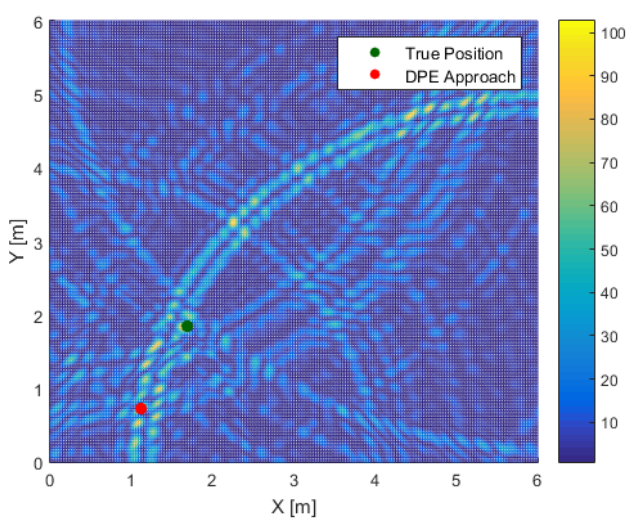

(a) Joint Periodogram realisation for DPE

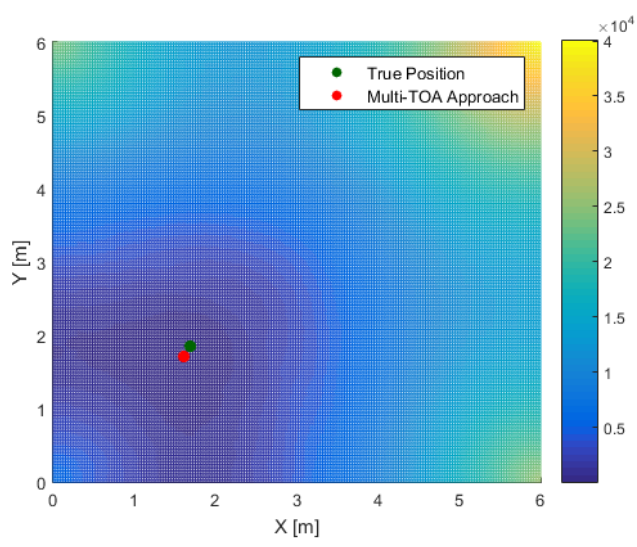

(b) Cost function for Multi-TOA

Fig. 2: Target localization results with 4 beacons.

\section{REFERENCES}

[1] S. Gezici, Z. Tian, G. B. Giannakis, H. Kobayashi, A. F. Molisch, H. V. Poor, and Z. Sahinoglu, "Localization via ultra-wideband radios: a look at positioning aspects for future sensor networks," IEEE signal processing magazine, vol. 22, no. 4, pp. 70-84, 2005.

[2] Z. Xiao, Y. Hei, Q. Yu, and K. Yi, "A survey on impulse-radio UWB localization," Science China Information Sciences, vol. 53, no. 7, pp. 1322-1335, 2010

[3] M. Navarro and M. Najar, "Frequency domain joint TOA and DOA estimation in IR-UWB," IEEE transactions on wireless communications, vol. 10, no. 10, pp. 1-11, 2011.

[4] O. Bialer, D. Raphaeli, and A. J. Weiss, "Efficient time of arrival estimation algorithm achieving maximum likelihood performance in dense multipath," IEEE Transactions on Signal Processing, vol. 60, no. 3, pp. 1241-1252, 2012.

[5] A. G. Amigó, P. Closas, A. Mallat, and L. Vandendorpe, "Cramér-Rao bound analysis of UWB based localization approaches," in 2014 IEEE International Conference on Ultra-WideBand (ICUWB). IEEE, 2014, pp. 13-18.

[6] E. Miljko and D. Vucic, "Direct position estimation of uwb transmitters in multipath conditions," in Ultra-Wideband, 2008. ICUWB 2008. IEEE International Conference on, vol. 1. IEEE, 2008, pp. 241-244.

[7] O. Bialer, D. Raphaeli, and A. J. Weiss, "Maximum-likelihood direct position estimation in dense multipath," IEEE Transactions on Vehicular Technology, vol. 62, no. 5, pp. 2069-2079, 2013.

[8] E. Tzoreff and A. J. Weiss, "Expectation-maximization algorithm for direct position determination," Signal Processing, vol. 133, pp. 32-39, 2017.

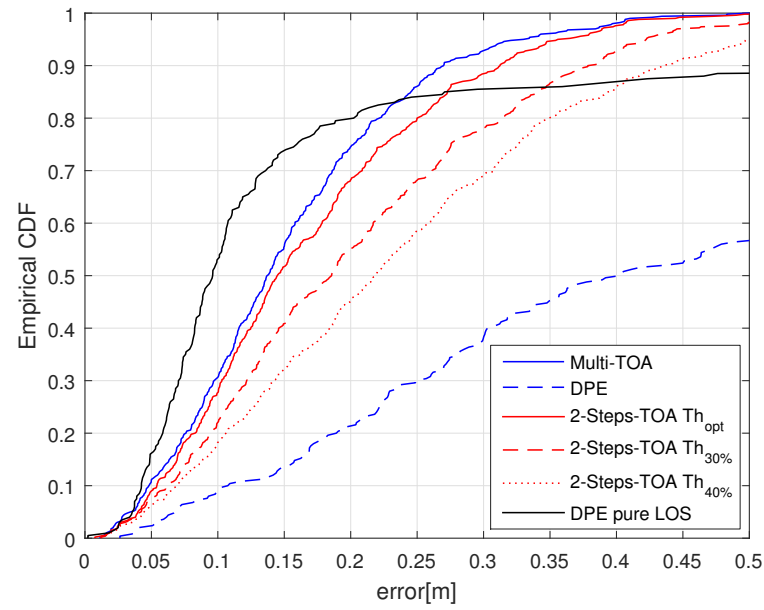

(a) Office LOS (CM3)

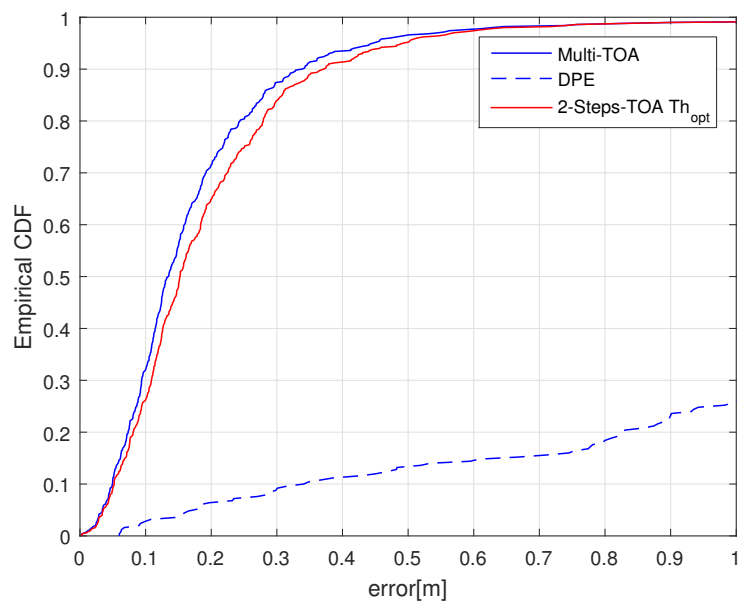

(b) Office NLOS (CM4)

Fig. 3: Empirical CDF of the position error using 4 beacons for Multi-TOA, DPE and 2-Steps-TOA.

[9] M. Navarro, P. Closas, and M. Nájar, "Assessment of Direct Positioning for IR-UWB in IEEE 802.15. 4a channels," in 2013 IEEE International Conference on Ultra-Wideband (ICUWB). IEEE, 2013, pp. 55-60.

[10] I. Guvenc and Z. Sahinoglu, "Threshold selection for UWB TOA estimation based on kurtosis analysis," IEEE Communications Letters, vol. 9, no. 12, pp. 1025-1027, 2005.

[11] D. Dardari, C.-C. Chong, and M. Win, "Threshold-based time-of-arrival estimators in UWB dense multipath channels," IEEE Transactions on Communications, vol. 56, no. 8, pp. 1366-1378, 2008.

[12] J. Clausen, "Branch and bound algorithms-principles and examples," Department of Computer Science, University of Copenhagen, pp. 1-30, 1999.

[13] A. F. Molisch, K. Balakrishnan, C.-C. Chong, S. Emami, A. Fort, J. Karedal, J. Kunisch, H. Schantz, U. Schuster, and K. Siwiak, "IEEE 802.15. 4a channel model-final report," IEEE P802, vol. 15, no. 04, p. 0662, 2004. 